\title{
Formal Modeling in a Commercial Setting: A Case Study
}

\author{
Andre Wong and Marsha Chechik \\ Department of Computer Science, University of Toronto, \\ Toronto, ON M5S 3G4, Canada, \\ \{andre, chechik\}@cs.toronto.edu, \\ http://www.cs.toronto.edu/ chechik
}

\begin{abstract}
This paper describes a case study conducted in collaboration with Nortel to demonstrate the feasibility of applying formal modeling techniques to telecommunication systems. A formal description language, SDL, was chosen by our qualitative CASE tool evaluation to model a multimedia-messaging system described by an 80 -page natural language specification. Our model was used to identify errors in the software requirements document and to derive test suites, shadowing the existing development process and keeping track of a variety of productivity data.
\end{abstract}

\section{Introduction}

For a long time, researchers and practitioners have been seeking ways to improve productivity in the software development process. Precise documentation of software specifications has been advocated as one of the viable approaches [25]. If high quality specifications are crucial to the success of system developments, it seems logical to apply rigorous specification techniques to the requirements for ensuring their completeness and consistency.

The majority of successful applications of formal modeling have been confined to safety-critical projects [5, 13, 19] where software correctness is the pivotal goal. In contrast, the commercial software industry seeks practical techniques that can be seamlessly integrated into the existing development processes and improve productivity; absolute quality is often a desirable but not crucial objective. Although the feasibility of formal specifications has been demonstrated in commercial settings [12, 15, 23], the overall adoption of the idea has been slow. Most companies, such as the Canadian-based telecommunications company Norte 1 , opt to rely on manual inspections of natural-language specifications as the only technique to detect errors in them, even though the results have been suboptimal. If the advantages of better quality specifications, such as a better understanding of the system and less error-prone designs, do not provide an adequate justification, more benefits can be obtained by leveraging the investment

\footnotetext{
${ }^{1}$ Nortel, for the purpose of this paper, refers to the Toronto Multimedia Applications Center of Nortel Networks.
} 
in the formalization process to other stages of the software lifecycle, i.e. generating code or test cases from the formal specifications. Not only does this amortize the cost of creating the specifications, but the productivity improvement can also be more immediate and easily measurable.

Driven by the need to shorten and improve the software development process, Nortel and the Formal Methods Laboratory at the University of Toronto have jointly proposed a pilot research project to investigate the feasibility of formal modeling techniques in a commercial setting. The goal of the project is to find means of using formal modeling to improve productivity in various stages of the software lifecycle in an economical manner. Specifically, the emphasis is placed on deriving test cases from the formal model as the Nortel engineers have expressed concerns about the feasibility of code generation for their proprietary platform.

Our exploratory project was organized as a hybrid quantitative and qualitative case study [21]. As it was extremely important to choose the right system/language combination for the formalization process, we began the study by selecting a typical system to specify and conducting a qualitative evaluation of formal modeling languages. A chosen language was applied to model the system, and the resulting model was used to identify errors in the software requirements document and to derive test suites, shadowing the existing development process. Throughout the study, we kept a variety of productivity data for comparison with similar information from the actual development process. We also noted the qualitative impact of the formalization process.

The rest of the paper is organized as follows: Section 2 provides a brief description of the software system selected for the study. Section 3 discusses the criteria used in choosing a suitable modeling language. In Section 4 we discuss the formalization process. Section 5 presents findings from the study. The experience gained during the project is summarized in Section 6 Section 7 briefly describes a usability workshop that we conducted at Nortel, and Section 8 concludes the paper.

\section{System Selection}

To make the project meaningful, we did not want to be directly involved in choosing a system, hoping to work on something representative of typical projects of the TorMAC division of Nortel. We also felt that it was important to do the formalization in parallel with the development cycle. Thus, a group of Nortel engineers, consisting of developers from the design team and testers from the verification team decided that we should work on a subsystem of the Operation, Administration and Maintenance (OAM) software of a multimedia-messaging system connected to a private branch exchange. The subsystem, called ServiceCreator in our paper, is a voice service creation environment that lets administrators build custom telephony applications in a graphical workspace by connecting predefined voice-service components together. Figure 1 illustrates the graphical view of one such telephony application consisting of four components: start, end, password-check, and fax. When the application is activated, a call session begins 
at the start component, and a caller is required to enter a numerical password in order to retrieve a fax document from the fax component. The caller is directed to the end component if an incorrect password is entered. In both scenarios, the call session ends when the end component is reached.

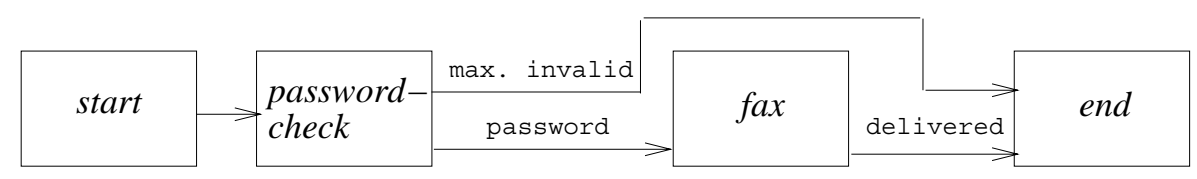

Fig. 1. A simplified telephony application example.

The lines connecting various components represent potential control flow of the call session, and the actions performed by the caller in an active component determine the output path taken. In the password-check component, for instance, the caller exits via the path password if a correct password is entered, or the path max. invalid if there are too many invalid password attempts.

In our study, we analyzed the run-time behavior of 15 such components, described by an 80-page natural language specification. We illustrate the approach using the password-check component, described by a 5-page natural language specification. Figure 2 shows a graphical view of this component.

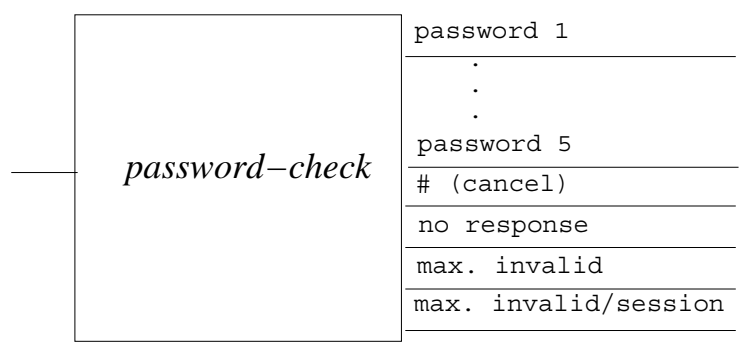

Fig. 2. The graphical view of the password-check component.

The purpose of this component is to validate digits entered by a caller against any of the passwords (up to five) defined by the administrator. For instance, the path password 1 is taken if the entered digits match the first defined password. The caller is forced to leave the component using the max. invalid output if the maximum number of invalid password attempts is reached. Such attempts are also monitored on a per-call session basis, and the caller leaves via the max. invalid/session output if the per-call session limit is reached. The caller can also enter the ${ }^{*}$ key to retrieve the help prompt, which has a side effect of clearing the password entry, or the \# key to exit prematurely via the \# (cancel) path if 
no password has been entered. If the caller stays idle for a certain time period and has not previously keyed in any digits prior to entering the password-check component, she is assumed to be using a rotary phone and is transferred to a different voice-service component. Otherwise, one of the two delay prompts may be played depending on whether she has begun keying in the password. After two more timeouts, the caller exits via the no response path.

In order to generalize from the results of the study, we need a good characterization of the type of applications that ServiceCreator represents. First of all, it is clearly a reactive system in the telecommunications domain. Additionally, it has relatively stable requirements and is fairly self-contained, having a loose coupling with the underlying system. Finally, it is not very complex although non-trivial.

\section{$3 \quad$ Evaluation of Modeling Methods}

A successful formalization of the system in a commercial setting depends crucially on a modeling language, supported by an appropriate tool. Thus, in this section we use the term method to indicate both the modeling language and its tool support. The goal of the evaluation was to select a suitable modeling method to be used in the feasibility study.

Easily readable and reviewable artifacts as well as a simple notation were the two basic requirements for a modeling method to be usable in a commercial setting. Moreover, since one of the overall objectives was to amortize the cost of creating a formal specification, we began the evaluation by conducting a broad survey [31] of available tools that provided support for both modeling and testing. These constraints turned out to be extremely limiting as most of the surveyed methods either had just the modeling or just the testing support, or did not have a formal notation. Some were simply too difficult to be used in industry. We eventually narrowed down our search to the following candidates:

- Telelogic SDT [27] - an integrated software modeling and testing tool suite that utilizes Specification and Description Language (SDL) [16], which is based on extended finite state machines (EFSMs), for behavioral modeling and Message Sequence Charts (MSCs) [17] for component-interaction specification. MSCs, which can be used as test cases, can be derived semiautomatically from an SDL model. Alternatively, SDT can verify the correctness of the model with respect to independently created MSCs.

- Aonix Validator/Req (V/Q) [2] - a test generation tool that allows blackbox requirements of a system to be specified in the form of parameterized UML [4] use cases. Validator/Req generates test cases for data-flow testing from the model automatically and produces documents that conform with the IEEE standard for software requirements specifications [1].

- Teradyne TestMaster (TM) [28] - a test generation tool that automatically generates test cases for both control-flow and limited data-flow testing from models based on EFSMs. The number of test cases can be flexibly tuned to suit different testing needs. 
To perform a detailed assessment, we structured our evaluation as a feature analysis exercise [21] and refined our focus to choosing among the remaining methods using additional evaluation criteria gathered from the Nortel engineers. These criteria comprised of factors, such as usability and smooth integration, that were crucial to the use of formal modeling in their environment. After the methods were used to model the password-check component, they were ranked against the criteria based on our impressions of the tool and the models produced.

\begin{tabular}{|l|c||c|c|c|}
\hline Criteria & Weighting & SDT & V Q & TM \\
\hline \hline has modeling support & 5 & 4 & 2 & 3 \\
\hline has testing support & 5 & 3 & 4 & 5 \\
\hline has a gentle learning curve & 4 & 3 & 5 & 3 \\
\hline produces easy-to-understand artifacts & 3 & 4 & 5 & 4 \\
\hline enhances understanding of the system & 4 & 5 & 3 & 4 \\
\hline $\begin{array}{l}\text { works smoothly with the existing development process } \\
\text { and tools }\end{array}$ & 4 & 5 & 4 & 3 \\
\hline $\begin{array}{l}\text { is scalable } \\
\text { has strong tool support }\end{array}$ & 3 & 4 & 2 & 3 \\
\hline performs completeness or consistency checks & 2 & 5 & 4 & 5 \\
\hline $\begin{array}{l}\text { provides features such as multi-user support, require- } \\
\text { ments traceability and document generation }\end{array}$ & 1 & 3 & 3 & 3 \\
\hline \hline Score & N/A & $\mathbf{1 3 7}$ & $\mathbf{1 2 1}$ & $\mathbf{1 2 4}$ \\
\hline
\end{tabular}

Table 1. Comparison of modeling methods.

Table 1 shows results of this evaluation. It lists the evaluation criteria in column one, their relative importance using a scale from 1 (least important) to 5 (most important) in column twd 2 , and the degree the methods satisfy the criteria using a scale from 1 (unsatisfactory) to 5 (completely satisfactory) in columns three to five. In the first row, for instance, SDL scores the highest as it allows the behavioral modeling and the hierarchical partitioning of a system into concurrent processes. TestMaster has similar modeling support but a system can only be modeled as a single hierarchical EFSM from a tester's perspective. Validator/Req ranks the lowest as its scenario-based notation provides very limited modeling support. The conclusion of the evaluation was reached by comparing the final scores for the methods obtained by adding weighted scores from each criterion, making SDT the most suitable tool.

A confounding factor in feature analysis is the potentially biased opinions of the evaluator. Although we tried to ensure the objectivity of the evaluation, assignments of the scores inevitably contained our subjective judgment, and we did not feel that we could accurately evaluate such factors as usability. To

$\overline{2}$ These were assigned after consultations with Nortel engineers. 
mitigate this potential problem and to gain more confidence in our assessment, we demonstrated the tools and the models to the engineers. They agreed that SDT satisfied their criteria more closely than the other tools.

\section{Modeling and Testing the System}

The next step was to formalize the ServiceCreator application. This formalization was undertaken by us in parallel with the actual development process. ServiceCreator was modeled as a 70-page SDL system in which the environment contained the underlying OAM software and messaging-system hardware. Out of the 15 voice-service components, 10 were modeled as separate SDL processes (see Appendix A for an example) that communicated with the environment through a "driver" SDL process. This process models the control-flow information of the telephone application. Figure 3 shows a simplified view of the "driver" process created for the application of Figure 1. This process is responsible for activating voice-service components and responding to their termination. The functionality of the five remaining components (e.g., start and end) was incorporated into the "driver" processes. A total of 23 signals were used in the SDL system; eight of them were external (used for communicating with the environment) and the rest internal. Persistent data such as the predefined passwords for the password-check component, were represented as parameters to the SDL processes.

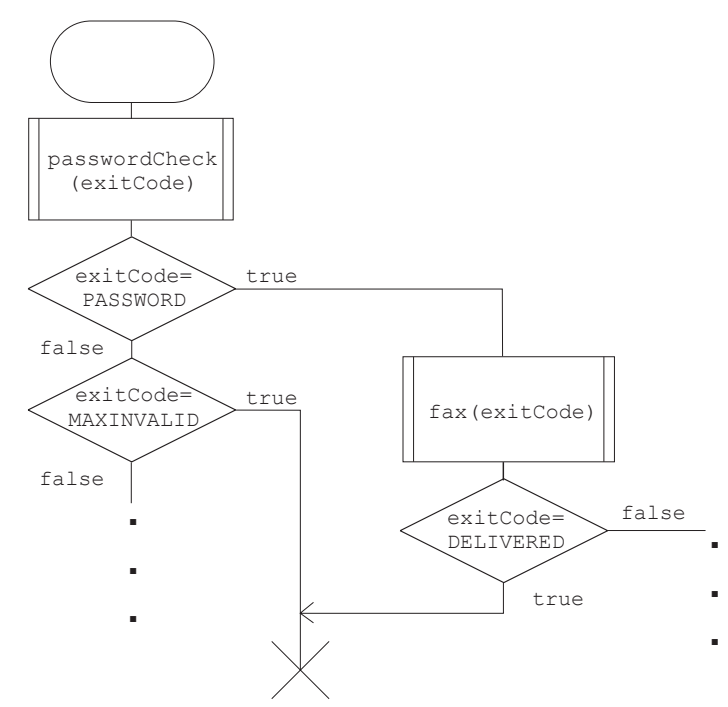

Fig. 3. A driver process for a simplified telephony application example of Fig. 1. 


\subsection{The Level of Abstraction}

The modeling process was relatively straightforward as we encountered no major problems in modeling ServiceCreator; we also felt that a background in formal methods was not required. However, the biggest concern was to determine the appropriate level of abstraction which was dictated by two opposing needs: the model should be constructed from a black-box view to reduce its complexity, while the exact behavior of the system needs to be modeled for deriving detailed test cases. In addition, a more detailed model would help in identifying problems in the natural-language specification.

Our approach was to start from a high level of abstraction, filling in details about some parts of the behavior if the natural-language specification required it. Mostly, such details were necessary in dealing with external input. For example, in modeling the password-check component, we represented the various timeouts by an SDL timer timeout (see Figure 4), as the actual length of the timeouts was relatively unimportant. Processing of user input, on the other hand, required reasoning on the level of single digits. In our model, a received digit, digit, was actually stored in a variable numberRecv. While this treatment could potentially lead to large and cluttered models, we sometimes had to resort to it to be able to derive sufficiently detailed test cases.

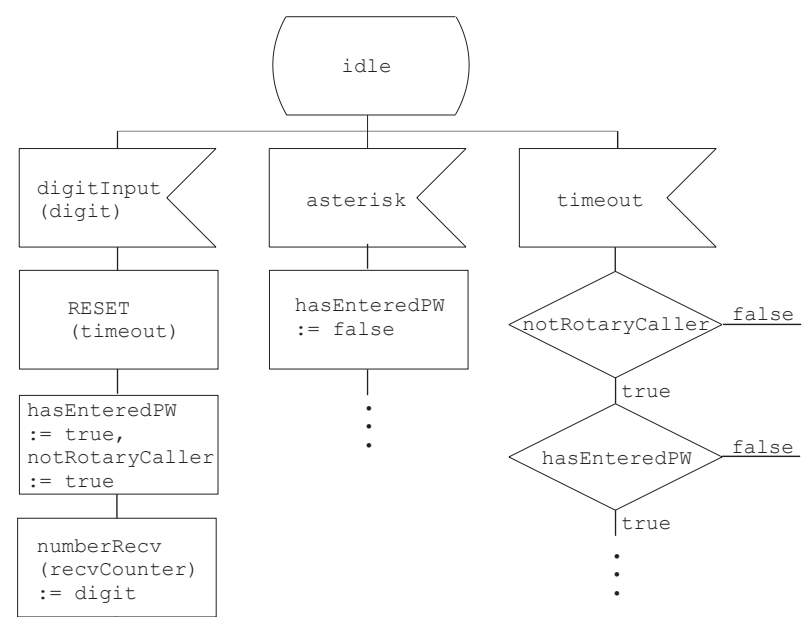

Fig. 4. A simplified fragment from the SDL model of the password-check component.

\subsection{Test Case Derivation}

To obtain immediate benefits from the formalization process, 120 MSCs were derived from the SDL model for testing the implementation. The derivation 
was not automatic: SDT recorded the interactions between the SDL system and its environment as MSCs when we animated the SDL model manually. We felt that the automation was not necessarily desirable since this exercise gave us confidence in the content and the coverage of the test cases.

During the test case derivation, we took advantage of the modular nature of the voice-service components and generated test cases for each of them separately, achieving full transition coverage in the corresponding SDL processes. However, some functionality of the system could be covered only by testing multiple components together, i.e. by integration testing. More than 20 integration test cases have been identified. For instance, to derive test cases for testing the initial timeout behavior for touch-tone callers where no passwords were keyed in, we created a telephony application model in which a caller was required to key in some digits in a component, such as a menu, prior to entering the passwordcheck component (see Appendix B for the corresponding MSC). The procedure for deriving such test cases was as follows:

- During the modeling phase, note the cases where the input comes not only from the environment but also from other components. If some input to component $A$ comes from component $B$, we say that there is a relationship between $A$ and $B$.

- After the modeling phase, use the resulting model to create test cases that specifically ensure that the relationship between the components is correctly implemented.

Derivation of test cases for integration testing was the most labor-intensive part of this phase; it also required a fair bit of skill.

\subsection{Specification and Implementation Problems}

The SDL model and the experience gained from the formalization process allowed us to identify specification errors that escaped a series of manual inspections by Nortel engineers. As the components modeled were not particularly complicated, most of the errors we found were caused by vagueness and missing information. In fact, the most time-consuming part of the formalization process was to understand the natural-language specifications and to consult the engineers for clarifications. We estimated that these activities took as much time as the formalization process. Some of the problems found in the specification of the password-check component are presented below:

1) it was never mentioned whether the various voice prompts were interruptible, and no conventions for such behavior were defined;

2) lengths of various timeouts were not mentioned;

3) it was unclear which exit path should be taken when the administrator set two or more passwords to be the same;

4) the maximum and minimum lengths of passwords were not defined. 
Some of these problems were very low criticality and could be easily fixed in the implementation. However, the testers were required to interact with the developers to clarify the correct behavior of the system, spending an unnecessarily long time in the test case execution phase (see Section 5.1). In addition, problem 4) propagated itself into the implementation. That is, a malicious caller could crash the system by entering an abnormally long password within the password-check component. Thus, this requirement omission became a critical implementation error. Moreover, since the MSCs derived from the SDL model were used to identify errors in the implementation after the Nortel engineers had officially completed testing of the voice-service components, we were able to observe that this implementation error had not been discovered. The reason was that the test cases were derived from the same incomplete specification and missed a critical error.

\section{Findings}

We began our analysis by seeking quantitative evidence to measure the effects of the formalization process on productivity. However, as the study progressed, we felt that it was also crucial to identify the qualitative factors (e.g., perceived usability of SDL and commitments from the development team) and the limitations of the study in order to reach accurate and unbiased conclusions.

Unfortunately, Nortel engineers did not keep track of many essential metrics and, due to the exploratory nature of our study, did not allow us to create a controlled environment where such metrics could be obtained. In particular, we do not know the exact amount of time it takes to fix a bug, if it is found during the inspection vs. design vs. testing phase. The lack of metrics significantly impairs our ability to draw quantitative conclusions.

\subsection{Quantitative Results}

The entire modeling process, which consisted of activities such as understanding and formalizing the specification as well as deriving and executing test cases, took about two person-months to complete. During this period, we kept track of a variety of productivity data in the study (column two of Table 2) for comparison with similar information from the existing development process (column three). Effort measurements in this table are approximated to the nearest person-day. Since the sizes of test cases varied greatly, we did not use a "test case" as the unit of comparison for testing-related data. Instead, we counted test units, the smallest externally visible functionality of the system, in each test case to ensure a fair comparison. Highlights from the table are summarized below:

- The time to model value included only the time used for modeling the SDL processes. While the modeling task did not have an equivalent in the actual development process, manual inspection was a similar activity that was also performed at the completion of the specification phase. Certainly, the formalization process was not meant to be a complete replacement. However, 


\begin{tabular}{|l||c|c|}
\hline Productivity Data & Study & Existing Process \\
\hline \hline Time to model (person-days) & 11 & N/A \\
\hline Time to inspect (person-days) & N/A & 50 \\
\hline Number of specification errors reported & 56 & N/A \\
\hline Number of test units & 269 & 96 \\
\hline Time to create test units (person-days) & 7 & 7 \\
\hline Time to execute test units (person-days) & 5 & 14 \\
\hline Number of implementation errors identified & 50 & 23 \\
\hline
\end{tabular}

Table 2. Productivity Comparisons.

if a large number of specification errors were identified in a relatively short amount of time, the modeling task could be considered a way to decrease the time for inspection. (We discuss this point later in this Section.)

- The number of specification errors reported could not be used for comparison as the Nortel engineers did not keep track of such statistics.

- More test units could be derived from the SDL model (which translated to better test coverage) in roughly the same amount of time, possibly because the model eased the creation task by providing a more in-depth understanding of the system as well as a better sense of completeness. One other reason for the difference in the quantity was that the test units from Nortel were sometimes vaguely specified (see Figure 5 for an example); the missing details contributed to a decrease in the number of test units reported.

Call the application and simulate a number of hacker attempts (such as common passwords and misspelled passwords). Verify that the application terminates the call session after the maximum number of password attempts is reached.

Fig. 5. A Nortel test case for testing the password-check component.

- The time needed for test unit execution in our study was much smaller for two major reasons: the derived test units were more detailed and thus easier to execute, and it was observed that Nortel engineers spent a lot of time revising the existing test cases because of the changes in requirements and creating more detailed test scenarios based on the vaguely specified test units. However, due to tight schedules, most testers did not document these extra units until the end of the entire testing phase, which spanned over more than four months. They admitted that some of these test units would inevitably be lost, contributing to a decrease in their total number.

- The number of implementation errors identified in the study was two times larger than that of the existing development process. Many of them were missed because testers created test cases from an incorrect and incomplete 
specification, as indicated in the third row of Table 2. Problems such as incompleteness propagate to the test cases and affect the test coverage. In fact, 18 of the 50 problems could be linked to problems in the specification. Their criticality ranged from the minor ones, dealing with the availability and the interruptability of voice prompts, to the critical ones, affecting the core functionality of the voice-service components or causing the system to crush. Most of these errors resulted from undocumented assumptions or incorrect/missing error handling.

To obtain an accurate cost/benefit figure, we needed to collect additional statistics such as an average cost to fix a requirements error discovered during the inspection and the implementation phases. As we mentioned above, the Nortel development team did not keep track of such metric; however, a conservative cost/benefit estimation is still possible. Without taking the improvement in software quality into account, we can estimate the cost of formalization by subtracting the time of the modeling task from the direct savings in

- the test unit creation (0 person-days),

- the test unit execution (9 person-days), and

- the manual inspection.

The formalization process did not include a manual inspection phase, whereas the actual development took 50 person-days for it. The modeling task would come at no cost if it were to reduce the manual inspection by 2 person-days, or $4 \%$. Of course, the actual cost/benefit figure is significantly more promising if the long-term benefits, coming from a better quality of the product, ease of maintenance and regression testing, and an ability to reuse a good specification, are taken into account.

\subsection{Qualitative Observations}

While all the quantitative data was in favor of the use of the formal modeling, it was clear that these results alone constituted only a part of our findings. Some observations from the formalization process that were not evident from Table 2 are discussed below.

- The most frequent complaint from the test engineers is that the missing information in the specifications often complicates the task of the test case creation. The SDL models encourage and assist developers in stating system requirements more precisely and completely, which should allow testers to create better quality (e.g., more detailed and with expected results more clearly defined) test cases and reduce the time needed for test case creation and execution. Developers should also benefit from the more complete specifications during the design and the implementation phases. This is an area where SDL can potentially significantly improve the development process. In fact, SDL has been successfully applied in the telecommunications field: from 
the traditional use of protocol specifications [3, 26, 8] to high level specification [22, prototyping [29], design [24], code generation [10], and testing [1] of telecommunications applications. Although the results reported in these studies were similar to ours, the goal of the studies was different: they were aimed to investigate technical advantages or a feasibility of SDL in a given environment, or were emphasizing only one of the development activities.

- As with any other formal specification technique, a successful integration of SDL into the development process requires a firm commitment from the entire development team. For instance, the developers must ensure that the SDL model is always kept consistent with the system requirements and the code, e.g. last-minute changes in the design and implementation are propagated back to the model. Testers also need to ensure that their test cases always reflect the model accurately. While this is possibly one of the biggest obstacles in applying a formal modeling technique, the advantages provided by SDL justify the extra effort.

- Compared to other formal modeling techniques, the strengths of SDL lie in its ease of use and the ability to express nontrivial behavior in a reviewable notation. Unlike many other formal modeling languages, SDL does not require an explicit use of formal logic. The graphical user-friendly notation allows developers without a strong mathematical background to effectively create EFSMs. Compared to natural language specifications, such EFSMs give a much better sense of completeness, allowing to easily detect missing scenarios, e.g., problem 1) in Section 4.3. In addition, the formal syntax helps clarify ambiguities or inconsistencies, e.g., problem 3) in the same section. However, SDL tends to blur the line between requirements and designs. If proper abstractions are not applied, the model may become too detailed and unnecessarily large, possibly duplicating the design effort.

\subsection{Limitations}

Based on the opinions expressed by the Nortel developers and testers, ServiceCreator was representative of the types of systems they have to work with, so we are fairly confident that the results of the study would apply to similar projects in this environment. We were also fortunate to find a method which is well suited for modeling telecommunication systems. However, it would be difficult [33] to generalize our findings outside the Nortel domain, since they would depend on the current development methodology, types of applications and the choice of a modeling language/tool.

Other limitations came from the fact that we had prior experience with SDL and were not constrained by development pressures. That is, we took the time necessary to produce high quality models and detailed test cases and felt that the process was straightforward. If time pressures prevent Nortel developers from applying the modeling techniques carefully, they may not achieve equally good results. In addition, novice users of SDL would take more time and possibly create less effective models of their systems. However, we believe that appropriate 
training and availability of an SDL expert can ensure that Nortel engineers use the SDL system successfully.

\section{Lessons Learned}

We were able to show that formal modeling techniques can shorten the development cycle while improving the quality of software. This can be done by amortizing the cost of creating the model over time-intensive phases of the software development lifecycle, such as testing or code generation. However, the total decrease in the development cycle is only achievable if the formalization can be done fairly inexpensively, by utilizing an easy-to-use and review notation, formalizing only selected components, and staying at a fairly high level of abstraction. It is also essential to achieve immediate results by using the approach incrementally, that is, being able to stop at any time and get partial benefits from partial modeling. A light-weight approach to formalization has been advocated by many researchers [6, 18, 20] and applied successfully in several projects, e.g. [7, 9].

What about verification? We feel that in the current commercial environment the majority of systems do not require any verification. There is typically a lesser need for absolute assurance, but a greater need for rapid development of reasonably correct systems. In fact, our use of SDL showed that, if verification is not involved, it is not essential to use a modeling language with a fully-defined formal semantics to achieve immediate and measurable benefits.

\section{Measuring Usability of SDL}

There is no doubt that usability of formal modeling techniques plays an important role in their acceptance in industry [14. An easy-to-use technique encourages experimentation and reduces the cost of integration. More importantly, the reality is that practitioners do not try to adapt to an inconvenient techniquethey simply abandon it 30]. Thus, it is essential that SDL is perceived to be usable by Nortel engineers. Only then will they be willing to apply it to their projects.

To collect some information about the usability of SDL, we conducted a oneday workshop in which six Nortel engineers participated. In the first part of the workshop we provided the participants with natural language descriptions of two small software systems 32 . After inspecting the descriptions manually and noting problems in them, the participants were asked to model the described systems in SDL. By formalizing the behavior, they were able to discover many additional specification errors; some of them found even more errors than we originally seeded, i.e. the descriptions contained errors that we did not notice. A few minor usability problems were noted, but the consensus reached among the participants was that the use of a formal, yet user-friendly, notation could help uncover problems hidden in the seemingly simple exercises much more effectively 
than manual inspections. In the second part of the workshop we asked the participants to fill in a questionnaire. The goal of the questionnaire was to obtain opinions about the usability of SDL and its perceived role in the development environment. Some of the results are summarized in Table 3. and the rest are available in 32 . The column on the right contains an average score on the scale from 1 (strongly disagree) to 5 (strongly agree).

\begin{tabular}{|l||c|}
\hline Statement & Score \\
\hline \hline SDL is easy to use & 3.7 \\
\hline $\begin{array}{l}\text { SDL can be used to address many of the development problems } \\
\text { we are facing. }\end{array}$ & 3.7 \\
\hline $\begin{array}{l}\text { The use of SDL increases our understanding of the requirements } \\
\text { and their quality. }\end{array}$ & 4.2 \\
\hline The use of SDL lengthens the requirements analysis phase. & 4.0 \\
\hline $\begin{array}{l}\text { The use of SDL shortens the design, implementation, and test- } \\
\text { ing phases. }\end{array}$ & 4.1 \\
\hline $\begin{array}{l}\text { Integrating SDL into my work routine is worthwhile and should } \\
\text { be tried. }\end{array}$ & 3.7 \\
\hline
\end{tabular}

Table 3. Some results from the questionnaire.

Results from the questionnaire strengthened our findings that SDL is a userfriendly formal modeling technique which can be used effectively by Nortel engineers to improve their development process. Encouraged by the prospects of SDL, Nortel and University of Toronto are in the process of setting up another joint project where the engineers will carry out the formalization process themselves, and we will only observe the progress and provide consulting, if necessary. Without many limitations of our study, this new project will provide a more accurate insight into the technical and economical feasibility of SDL in a commercial setting.

\section{Conclusions}

In this case study we formalized the behavior of a multimedia-messaging system in a commercial setting. The success of the study was in finding a representative system, carefully selecting a suitable modeling method, and taking a lightweight formalization approach. Although we did not have access to some development metrics to fully quantify our findings, the results of the study clearly show that software requirements can be formalized effectively and economically, yielding significant improvements over the existing development process.

\section{Acknowledgments}

The authors would like to thank Albert Loe, Steve Okun, Avinash Persaud, and Shawn Turner of Nortel for their technical assistance and continual support 
throughout the study. We are also grateful to the anonymous referees for suggesting ways to improve the paper. The study was supported in part by Nortel Networks, NSERC, and Ontario Graduate Scholarship (OGS).

\section{References}

[1] ANSI/IEEE. "IEEE 830: IEEE Recommended Practice for Software Requirements Specifications ". IEEE, 1993.

[2] Aonix. "Aonix Home Page". http://www.aonix.com, September 1998.

[3] F. Belina, D. Hogrefe, and A. Sarma. SDL with Applications from Protocol Specification. Prentice Hall, 1991.

[4] UML Partners Consortium. "UML Proposal Document Set". OMG documents ad/97-08-\{02,03,04,05,06,07,08,09\}, September 1997.

[5] J. Crow and B.L. Di Vito. "Formalizing Space Shuttle Software Requirements". In Workshop on Formal Methods in Software Practice, San Diego, California, January 1996.

[6] R.E. Davis and R.L. Danielson. "Practically Formal Methods". In Proceedings of International Conference on Software Engineering: Education and Practices, pages 168-175. IEEE Computer Society Press, January 1996.

[7] Steve Easterbrook, Robyn Lutz, Richard Covington, John Kelly, Yoko Ampo, and David Hamilton. "Experience Using Lightweight Formal Methods for Requirements Modeling". IEEE Transactions on Software Engineering, 24(1):4-14, January 1998.

[8] Christian Facchi, Markus Haubner, and Ursula Hinkel. The SDL Specification of the Sliding Window Protocol Revisited. Technical Report TUM-I9614, Technische Univerität München, 1996.

[9] M.S. Feather. "Rapid Application of Lightweight Formal Methods for Consistency Analysis". IEEE Transactions on Software Engineering, November 1998.

[10] M.W. Froberg. "Automatic Code Generation from SDL to a Declarative Programming Language". In Proceedings of the Sixth SDL Forum, Darmstadt, Germany, October 1993.

[11] M. Grasmanis and I. Medvedis. "Approach to Behaviour Specification and Automated Test Generation for Telephone Exchange Systems". In Proceedings of the Fifth SDL Forum, Glasgow, Scotland, September 1991.

[12] Anthony Hall. "Using Formal Methods to Develop an ATC Information System". IEEE Software, 13(2), March 1996.

[13] Mats P.E. Heimdahl. "Lessons from the Analysis of TCAS II". In Proceedings of the International Symposium on Software Testing and Analysis (ISSTA'96), San Diego, CA, January 1996.

[14] Constance Heitmeyer. "One the Need for Practical Formal Methods". In Proceedings of 5th Int. Symp. on Real-time and Real-time Fault Tolerant Systems (FTRTFT'98), pages 18-26, 1998. LICS 1486.

[15] Jonathan P. Hoare. "Application of the B-Method to CICS". In M. G. Hinchey and J. P. Bowen, editors, Applications of Formal Methods, pages 97-124. Prentice Hall International Series in Computer Science, 1995.

[16] ITU-T. "ITU-T Recommendation Z.100: Specification and Description Language (SDL)". ITU-T, 1993.

[17] ITU-T. "ITU-T Recommendation Z.120: Message Sequence Chart (MSC)". ITUT, 1993. 
[18] Daniel Jackson and Jeannette Wing. "Lighweight Formal Methods". IEEE Computer, April 1996.

[19] Paul K. Joannou. "Experiences from Application of Digital Systems in Nuclear Power Plants". In Proceedings of the Digital Systems Reliability and Nuclear Safety Workshop, Rockville, Maryland, 1993.

[20] Cliff B. Jones. "An Invitation to Formal Methods: A Rigorous Approach to Formal Methods". IEEE Computer, 20(4):19, April 1996.

[21] Barbara Ann Kitchenham. "Evaluating Software Engineering Methods and Tools. Part 1". ACM SIGSOFT Software Engineering Notes, 21(1):11-15, January 1996.

[22] L. Mansson. "High Level Specification of a Telecom Application with SDL'92". In Proceedings of the Sixth SDL Forum, Darmstadt, Germany, October 1993.

[23] Peter Mataga and Pamela Zave. "Multiparadigm Specification of an AT\&T Switching System". In M. G. Hinchey and J. P. Bowen, editors, Applications of Formal Methods, pages 375-398. Prentice Hall International Series in Computer Science, 1995.

[24] M.Kooij and L. Provoost. "Industrial Report on the Use of Abstraction in SDL/MSC". In First Workshop of the SDL Forum Society on SDL and MSC, Berlin, Germany, June 1998. alcatel.

[25] D.L. Parnas. "Some Theorems We Should Prove". In Proceedings of 1993 International Conference on HOL Theorem Proving and Its Applications, Vancouver, BC, August 1993.

[26] A. Sarma. "Modelling Broadband ISDN Protocols with SDL". In Proceedings of the Fifth SDL Forum, Glasgow, Scotland, September 1989.

[27] Telelogic. "Telelogic SDT Home Page". http://www.telelogic.com/solution/ tools/sdt.asp, September 1998.

[28] Teradyne. "TestMaster Home Page". http://www.teradyne.com/prods/sst/ ssthome.html, September 1998.

[29] H.J. Vgel, W. Kellerer, S. Karg, M. Kober, A. Beckert, and G. Einfalt. "SDL based prototyping of ISDN-DECT-PBX switching software". In First Workshop of the SDL Forum Society on SDL and MSC, Berlin, Germany, June 1998.

[30] Debora Weber-Wulff. "Selling Formal Methods to Industry". In J.C.P. Woodcock and P.G. Larsen, editors, Proceedings of FME'93: Industrial Benefit of Formal Methods, First International Symposium of Formal Methods Europe, pages 671678, Odense, Denmark, April 1993. Springer-Verlag.

[31] Andre Wong. "The Diary of the Formal-Method Survey". http://www.cs. toronto.edu/ andre/progress.html, September 1998.

[32] Andre Wong. "Formalizing Requirements in a Commercial Setting: A Case Study". M.Sc. thesis, University of Toronto, Department of Computer Science, Toronto, ON, Canada, 1999. (In preparation).

[33] Marvin V. Zelkowitz and Dolores R. Wallace. "Experimental Models for Validating Technology". IEEE Computer, 31(5), May 1998. 


\section{A An SDL Block Diagram}

The diagram below illustrates the SDL block diagram of the telephony application shown in Figure 1. The "driver" block (which contains a "driver" process) acts as the signal router between the environment and the SDL blocks of the voice-service components by routing the signal lists inputSigLst and outputSigLst. It is also responsible for activating the appropriate component according to the control-flow of the application and actions from the caller by using the signal activate. Please refer to Section 4 for more details.

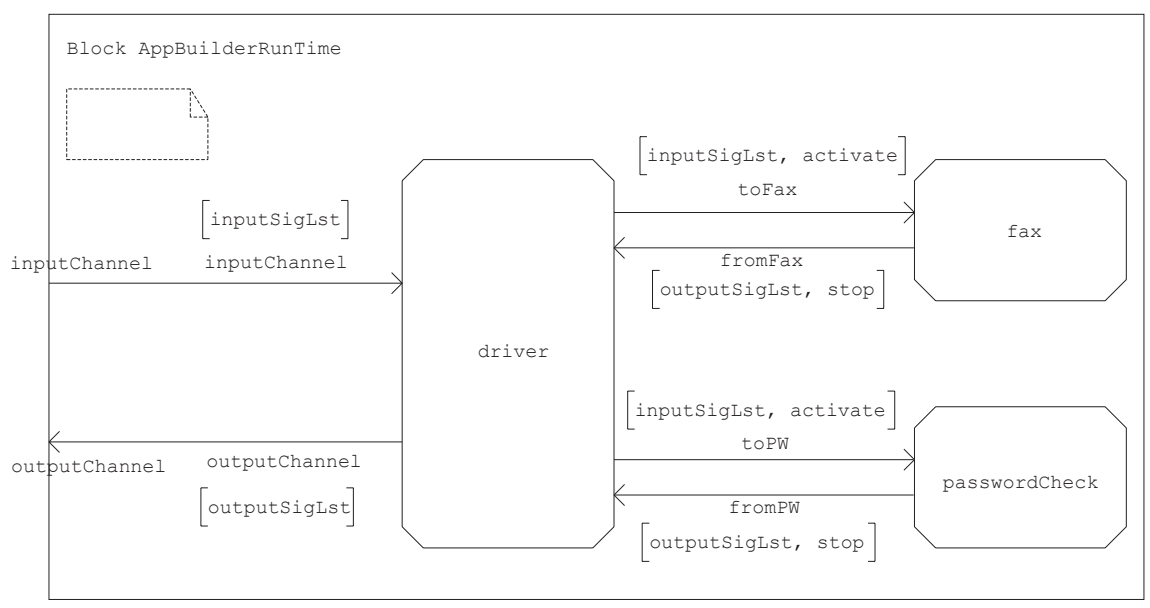




\section{B A Message Sequence Chart}

The MSC below shows the interactions between a caller and a telephony application in ServiceCreator. The application requires a caller to press button 1 in the menu component prior to entering the password-check component. Refer to Section 4.2 for more details.

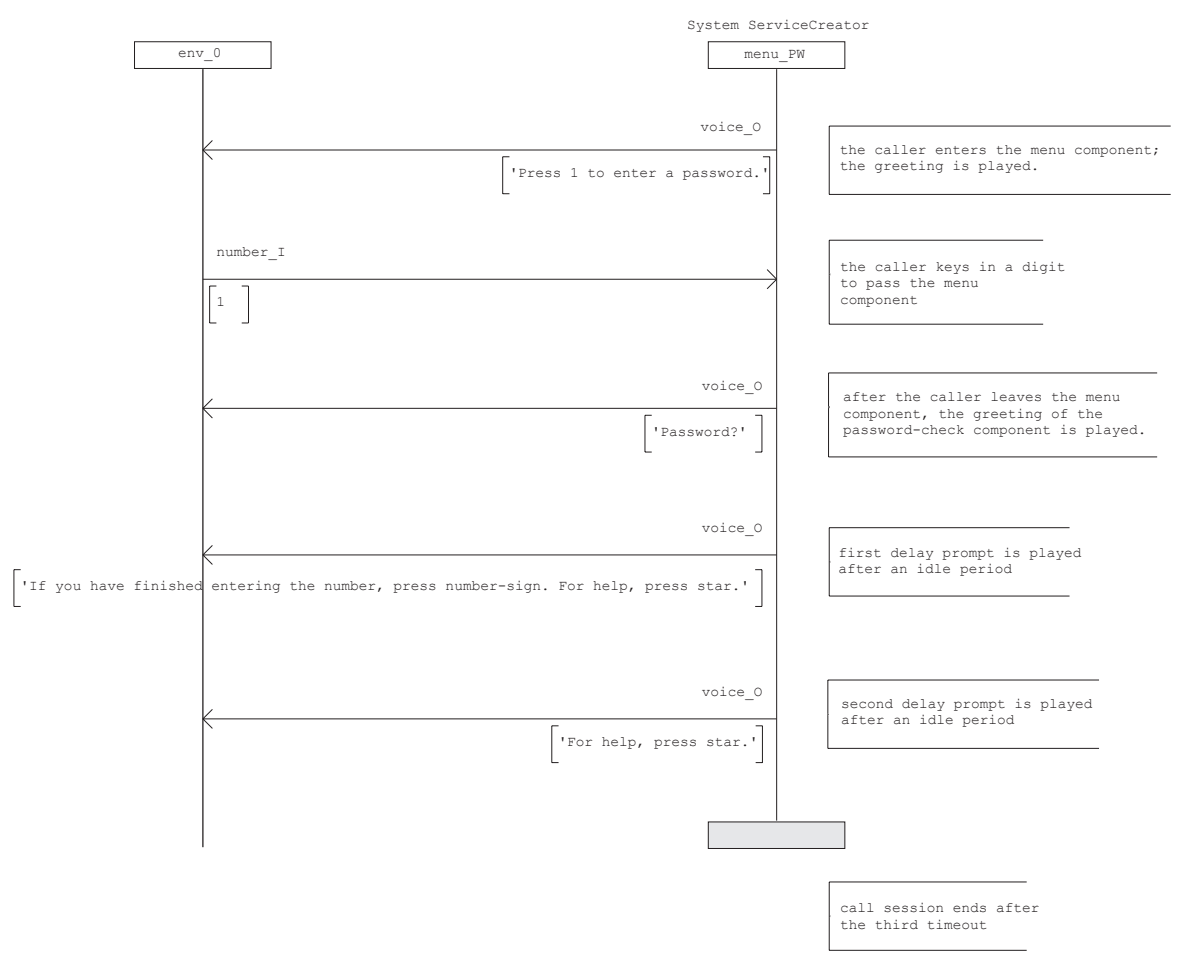

